\title{
Factors affecting the value of a simple biochemical scheme for identifying Enterobacteriaceae: the reproducible recognition of biotypes
}

\author{
J. G. BARR, REBECCA J. MAHOOD, AND K. P. W. CURRY \\ From the Department of Clinical Bacteriology, Royal Victoria Hospital, Belfast, Northern Ireland
}

SUMMARY A biochemical screening scheme on agar media for differentiating Enterobacteriaceae in a hospital laboratory is evaluated. Careful observation of test reactions within the scheme permitted the recognition of 78 biotypic reaction patterns which could contribute to epidemiological surveillance. The limitations of the technique are described and discussed and methods of importance in ensuring the reproducibility of reaction patterns emphasised.

Many workers have described biochemical schemes for identifying Enterobacteriaceae (Carpenter et al., 1966; Rosner, 1970; Chadwick et al., 1974; Lassen, 1975) and several commercially available systems have been evaluated (Tomfohrde et al., 1973; Nord et al., 1975).

The scheme described here is based on biochemical tests which have been shown over a long period to give reproducible and clear results. The small and specific number of tests chosen was based on our experience of the relative occurrence of genera and species and designed to allow the simplest identification scheme for the Enterobacteriaceae commonly isolated in this laboratory. Other evidence shows that this distribution of isolates is not uncommon in other routine medical laboratories (Rosner, 1970; Berger and Einecke, 1973; Chadwick et al., 1974).

The tests adopted included many of those considered by Chadwick et al. (1974) to be valuable in the definition of the major taxa proposed by Edwards and Ewing (1962). The value of aesculin hydrolysis (Wasilauskas, 1971; Barr and Mahood, 1977) and inositol fermentation (Donovan, 1966; Barr and Mahood, 1976) in differentiating the KlebsiellaEnterobacter-Serratia group from other Enterobacteriaceae has already been established, and Proteus spp and Providencia spp are differentiated from other Enterobacteriaceae and from one another on the basis of phenylalanine deaminase and urease activity. Other tests, including tryptophanase activity, growth in presence of potassium cyanide, citrate

Received for publication 29 November 1976 utilisation, and colony colour and hydrogen sulphide production on xylose-lysine deoxycholate medium (XLD medium; Taylor, 1969), contributed to the differentiation of Citrobacter spp and Escherichia coli and aided differentiation among other genera. Ornithine decarboxylase activity differentiatesEnterobacter spp and Serratia spp from other Klebsielleae and aids differentiation between Proteus spp. These tests have allowed a differentiation of the vast majority of clinical isolates of Enterobacteriaceae into the major taxa described by Edwards and Ewing (1962).

It should be noted that variations in either the rate or degree of specific enzyme action may allow differentiation between genera or between species in a standardised system. Thus the degree of aesculin hydrolysis and inositol fermentation may indicate species or biotype differences among Klebsielleae (Barr and Mahood, 1976).

This system, by highlighting unusual biotypic reaction patterns of individual genera or species, may often allow recognition of patterns of biochemical results which may be of value in epidemiological studies. This may be recognised in the incidence of tryptophanase-positive isolates of Enterobacter spp, Klebsiella aerogenes, and Proteus mirabilis and by variations in production of hydrogen sulphide by $\boldsymbol{P}$ mirabilis and Citrobacter spp on XLD and inositol-hydrogen sulphide-motility media.

In these laboratories we have tried to demonstrate the importance of inoculum size, incubation time, and reproducibility in the semi-quantitative interpretation of individual biochemical results on the performance of the scheme. It should not be 
assumed that strictly positive or strictly negative biochemical results are the only viable and reproducible alternatives when standardised methods are adopted.

\section{Material and methods}

\section{ORGANISMS}

\section{Clinical isolates}

A total of 1659 clinical isolates were biotyped by the scheme described over a period of eight months. These comprised 1197 cultures derived from urinary tract infections and a further $\mathbf{4 6 2}$ isolations derived from other sources in the routine bacteriological laboratory.

\section{Stock cultures}

The 30 stock cultures listed below were supplemented with 25 cultures of Enterobacteriaceae previously isolated from clinical material, stored, and their identification authenticated by extensive biochemical testing and by other workers. The National Collection of Type Cultures (NCTC) cultures examined were Klebsiella aerogenes (2 strains) NCTC 8172 and 9668; $K$ altantae NCTC 9496; $K$ edwardsii NCTC 5054; K pneumoniae (4 strains) NCTC 9633, 204, 8532 , and 10246; $K$ ozaenae (4 strains) NCTC 5050 , 9659,10313 , and $8883 ; K$ rhinoscleromatis (2 strains) NCTC 5046 and 5047; Enterobacter aerogenes NCTC 10006; E cloacae NCTC 10005; Serratia marcescens NCTC 9940; Escherichia coli (2 strains) NCTC 10418 and 9001; Proteus mirabilis NCTC 5887; $P$ morganii NCTC 235; $P$ vulgaris NCTC 4175; $P$ rettgeri NCTC 7475; Providencia $B$ subgroup $B$ NCTC 10318; Providencia spp NCTC 1026; Citrobacter freundii (2 strains) NCTC 9750 and 8090; Yersinia enterocolitica NCTC 10460; and Pseudomonas aeruginosa NCTC 10332 and 10701.

\section{CULTURE MEDIA}

Aesculin bile agar (Difco), Simmon's citrate agar (Difco), phenylalanine agar (Difco), and xylose lysine deoxycholate agar (Difco) were utilised as described by Chadwick et al. (1974).

Ornithine decarboxylase agar, derived from the fluid medium of Fay and Barry (1972), was prepared in $1.5 \%$ agar (Oxoid L11). The inositol-hydrogen sulphide-motility medium and potassium cyanide medium were prepared as described by Barr and Mahood (1977). Urease medium was that proposed by Christensen (1946) with the modifications described by Cowan (1974).

Replidishes (Sterilin Ltd) were used throughout, $3 \mathrm{ml}$ of each medium, except inositol-hydrogen sulphide-motility medium $(4 \mathrm{ml})$ being dispensed in each compartment.

For standard cultures and 50 clinical isolates biochemical tests were also carried out by classical methods as described by Cowan (1974).

Bacterial isolates not identified on screening media were identified after further tests-namely, arabinose fermentation ( $1 \%$ peptone water); lysine decarboxylase activity (Decarboxylase base (Difco) according to Moller, 1955), and methyl-red and VogesProskauer tests (Cowan, 1974, using the method described by Barritt, 1936).

The quality of all screening media was controlled by regular testing with standard cultures of known reaction.

PREPARATION OF INOCULA

Inocula for screening media were prepared by trans- 을 ferring at least one colony from a primary isolation plate into peptone water and incubating at least 4 hours at $37^{\circ} \mathrm{C}$ to obtain a culture of optical density $\overparen{D}$ $0 \cdot 2-0.5$ at $700 \mu \mathrm{m}$ as measured by a Hilger spectro- $\vec{\varphi}$ photometer. A practised eye can quickly judge a $\forall$ turbid culture to be within or significantly below this range of optical density.

Sequential tenfold dilutions of this inoculum were prepared in $9 \mathrm{ml}$ distilled water and estimates of total viable cells calculated by the method of Miles and Misra (1938).

Inositol-hydrogen sulphide-motility medium was inoculated using a sterile straight platinum wire. Other media were inoculated with one drop, about $0.02 \mathrm{ml}$, of peptone water culture or a derived dilution from a sterile Pasteur pipette.

\section{CONDITIONS OF INCUBATION}

The peptone water culture used for inoculation of $O$ agar media was reincubated for a further 16 hours and used for detecting tryptophanase activity.

All media were incubated at $37^{\circ} \mathrm{C}$ for 16 hours with $D$ lids of replidishes placed in the vented position. Before incubation ornithine decarboxylase agar was $N$ overlaid with sterile liquid paraffin $(0.5 \mathrm{ml}$ per compartment). Urease agar plates were sealed with 0 one-inch wide adhesive cellophane tape strips $\omega$ (Sellotape) and placed in parallel in such a manner? that two strips overlaid each compartment junction. Contact between sealing tape and replidish was obtained by gentle finger pressure, preventing? diffusion of volatile metabolites from one compartment to another.

RECORDING BIOCHEMICAL REACTIONS Citrate utilisation was recorded by indicator colour change or growth, or both; ornithine decarboxylase 
activity by indicator colour change; resistance to potassium cyanide by presence or absence of growth; and indole production detected by use of Kovac's reagent. Colony colour on XLD medium was recorded and the presence or absence of medium blackening due to hydrogen sulphide production noted. In inositol-hydrogen sulphide-motility medium hydrogen sulphide production was recorded by sulphide precipitation after 16 hours. Motility, made visible by reduction of triphenyltetrazolium chloride to formazan, was recorded after 16 hours.

Urease activity was recorded according to indicator colour change as strong $(3+)$, characteristic of Proteus spp; moderate $(2+)$, characteristic of Klebsiella spp; weak $(1+)$, characteristic of Enterobacter spp and Yersinia enterocolitica; and absent (-).

Aesculin hydrolysis and inositol fermentation were recorded in a semi-quantitative manner as previously described (Barr and Mahood, 1976, 1977). The degree of aesculin hydrolysis was recorded as strong $(2+)$, complete colour change within inoculation compartment; weak $(1+)$, hydrolysis in the immediate area of bacterial growth; and (-) no hydrolysis. Inositol fermentation was recorded as $(3+)$, complete indicator colour change within inoculation compartment; $(2+)$, distinct zone of indicator colour change along line of stab inoculation, and $(-)$ in the absence of fermentation.,

To ascertain the effect of inoculum size on test results on screening media the response of tenfold serial dilutions of bacteria representative of the major genera and species were evaluated by the methods described above.

IDENTIFICATION OF CLINICAL ISOLATES

Bacteria were identified as far as possible on the basis of results obtained on screening media. However, in an exploratory study with culture collection bacteria and 50 clinical isolates of Enterobacteriaceae biochemical tests were also carried out in parallel in classical fluid media. When these tests failed to give species identification (or with Citrobacter genus definition) bacteria were further tested and identified according to the methods described by Cowan (1974).

In the subsequent study isolates which on screening media alone failed to allow species identification were further tested by methyl-red Voges-Proskauer, arabinose fermentation, and lysine decarboxylase tests. Routinely no attempt was made by further biochemical testing to differentiate between Klebsiella aerogenes, $K$ edwardsii, and $K$ atlantae; to delineate species among Citrobacter isolates; or to give species definition to atypical Proteus isolates.

\section{Results}

COMPARISON OF BIOCHEMICAL TESTING BY SCREENING MEDIA AND BY CLASSICAL METHODS

The biochemical reactions of stock cultures and 50 clinical isolates were compared on screening media and classical test media.

Reactions obtained for ornithine decarboxylase activity, inositol fermentation, and aesculin hydrolysis were identical by both methods. It was possible also, as in the screening media, to distinguish between the aesculin reaction of Pseudomonas spp and Klebsielleae on agar slopes. Similarly those bacteria showing late fermentation (2-6 days) in inositol peptone water corresponded with those showing only trace fermentation along the line of stab inoculation on the screening medium.

Identical results were also derived from phenylalanine deaminase and urease activity, although it was impossible on slope media to distinguish between the intensity of Proteus spp and Klebsiella spp urease activity. Contrary to the observations of Chadwick et al. (1974), phenylalanine deaminase activity on the screening medium was demonstrated for all isolates of Proteus morganii.

Some variation in results obtained in 16-hour incubations were, however, observed with potassium cyanide $(\mathrm{KCN})$ and Simmon's citrate media with stock cultures. Errors in determination of $\mathrm{KCN}$ resistance were often observed in our use of $\mathrm{KCN}$ broth (Rogers and Taylor, 1961) and we had much less difficulty in observing growth on an agar base. With Simmons's citrate medium the divergence in results was due to difficulties in discerning, after 16 hours' incubation, growth or indicator colour change in citrate agar distributed in Bijou bottles. Those cultures which, in conflict with screening medium results, were recorded as negative after 16 hours' incubation were always recorded as positive after 24-72 hours. No positive citrate result was encountered in Bijou bottles which was not accompanied by a similar result on the screening medium.

In summary, we may say that divergence of results resided mainly in the easier observation of reactions in media distributed in replidishes (Sterilin).

Repeated screening of stock cultures demonstrated the reproducibility of test medium results and therefore the biotype reaction patterns recorded. Reproducibility was further established when multiple isolates from one source were shown to give the same biotypic reaction pattern.

EFFECT OF INOCULUM SIZE ON RESPONSE PRODUCED ON SPECIFIC SCREENING MEDIA The primary aim of this investigation was to establish 
conditions under which reproducible reaction patterns for bacterial isolates would be obtained from repeated isolations of the same bacterium or from repeated biochemical testing of the same isolate.

Conditions of medium preparation, medium dispensing, and test incubation conditions were readily standardised. However, since it was more difficult to standardise inoculum size, the effect of variations in inoculum size on specific test results during 16-hour incubations was evaluated. Each test medium was evaluated in turn in the presence of dilutions of organisms which gave known responses on those media (Table 1).

A number of screening media, XLD agar, phenylalanine deaminase agar, and peptone waterused for detecting tryptophanase activity-are not included. In all cases these 3 tests gave the same result irrespective of inoculum size. Other media, clearly influenced by inoculum size, are considered in turn.

The rate of aesculin hydrolysis by Klebsielleae has already been shown to be more rapid than that of non-Klebsielleae (Barr and Mahood, 1977). The investigations described here show that while large variations in inoculum size have little effect on the reaction demonstrated by Klebsielleae reduction in inoculum size of aesculin hydrolysing isolates of $E$ coli may result in the recording of erroneous negative results after 16-hour incubations. No differentiation between aesculin hydrolysis of $K$ aerogenes and $E$ aerogenes was recorded.

The detection of citrate utilisation as a sole carbon source was, as described earlier, easier in screening media than by conventional test methods. Only with Proteus mirabilis was the effect of inoculum size considered to be significant in screening media. As shown in Table 1, sequential reductions in inoculum size resulted in increasingly reduced detection of citrate utilisation in 16-hour incubations. This observation should not result in difficulties in differentiation between Proteus vulgaris and $P$ mirabilis but may certainly be an important factor in the differentiation of some intermediate biotypes of $\boldsymbol{P}$ vulgaris and $\boldsymbol{P}$ mirabilis.

In the scheme adopted here considerable dependence has been placed on accurate demonstration of ornithine decarboxylase activity in differentiating Klebsiella spp from Enterobacter spp and Serratia spp and in species differentiation among Proteus isolates. It is clear from the results given in Table 1 that a reduction in inoculum size of Enterobacter aerogenes from $10^{8}$ to $10^{6}$ viable cells $/ \mathrm{ml}$ would significantly reduce the detection of this enzyme and could render some isolates indistinguishable from Klebsiella spp. While detection of ornithine decarboxylase activity by other isolates is unlikely, for the most part, to influence species identification, variation in inoculum size over a narrow range would play a considerable part in differentiating biotypes of $E$ coli and Proteus morganii.

This limited investigation showed that for most genera reduction in inoculum size over a considerable range did not influence the recording of sensitivity or resistance to potassium cyanide. The most important differentiation of Citrobacter spp and $E$ coli was not influenced by inoculum size within normal ranges. The variation due to inoculum size among the Klebsielleae is unlikely in the screening regimen employed to influence Klebsielleae recognition, although possible error on recognition of $K$ pneumoniae is noted in the sensitivity of $K$ aerogenes to $\mathrm{KCN}$ when low inocula were used.

Motility was not influenced by inoculum size of except among isolates of $E$ coli. Although non-motile 을 isolates of $E$ coli are not infrequent their pre- dominance among those screened in this study $c$ (Table 2) is more likely to be due to slight differences $\underset{\bar{D}}{\overrightarrow{0}}$ in inoculum size among those screened than to a preponderance of non-motile isolates. Although we have not checked this observation the results shown in Table 1 demonstrate that recognition of motility in the screening media by $E$ coli is more sensitive to inoculum size than other genera investigated. In view of the sensitivity to inoculum size of ornithine decarboxylase activity among Enterobacter isolates it is of value to note here that motility of Entero- $\overrightarrow{\bar{a}}$ bacter aerogenes was normally recorded in this 3 medium and should allow adequate differentiation from Klebsiella spp in the screening scheme.

Among Citrobacter spp and Proteus spp examined hydrogen sulphide production by Proteus morganii ${ }_{0}$ alone was shown to be sensitive to inoculum size 3 (Table 1). Since biotypes have been encountered among Citrobacter spp and Proteus spp (Table 2) which failed to produce hydrogen sulphide under the 0 experimental conditions employed, the insensitivity of isolates reported here to reduction in inoculum size is considered to be valuable in the distinction of biotypes within these genera.

Proteus spp always demonstrated strong $(3+) \mathrm{N}$ urease activity and could therefore be readily N differentiated from Providencia stuartii, irrespective $\omega$ of inoculum size. With high inocula Klebsiella aerogenes/oxytoca isolates all gave a strong urease reaction, sufficient to differentiate them from isolates $\stackrel{\Phi}{-}$ of Enterobacter spp. The medium and experimental 0 conditions here may also, as suggested by Chadwick et al. (1974), differentiate isolates of Yersinia $\vec{\otimes}$ enterocolitica from $E$ coli. The single isolate of $\frac{}{\mathbb{D}}$ $Y$. enterocolitica evaluated here demonstrated urease $\frac{\varrho}{\sigma}$ activity over a wide range of inoculum size.

The rate of inositol fermentation by non-Kleb-ᄋ 
Table 1 Effect of inoculum size on results recorded by different species or genera on various screening media

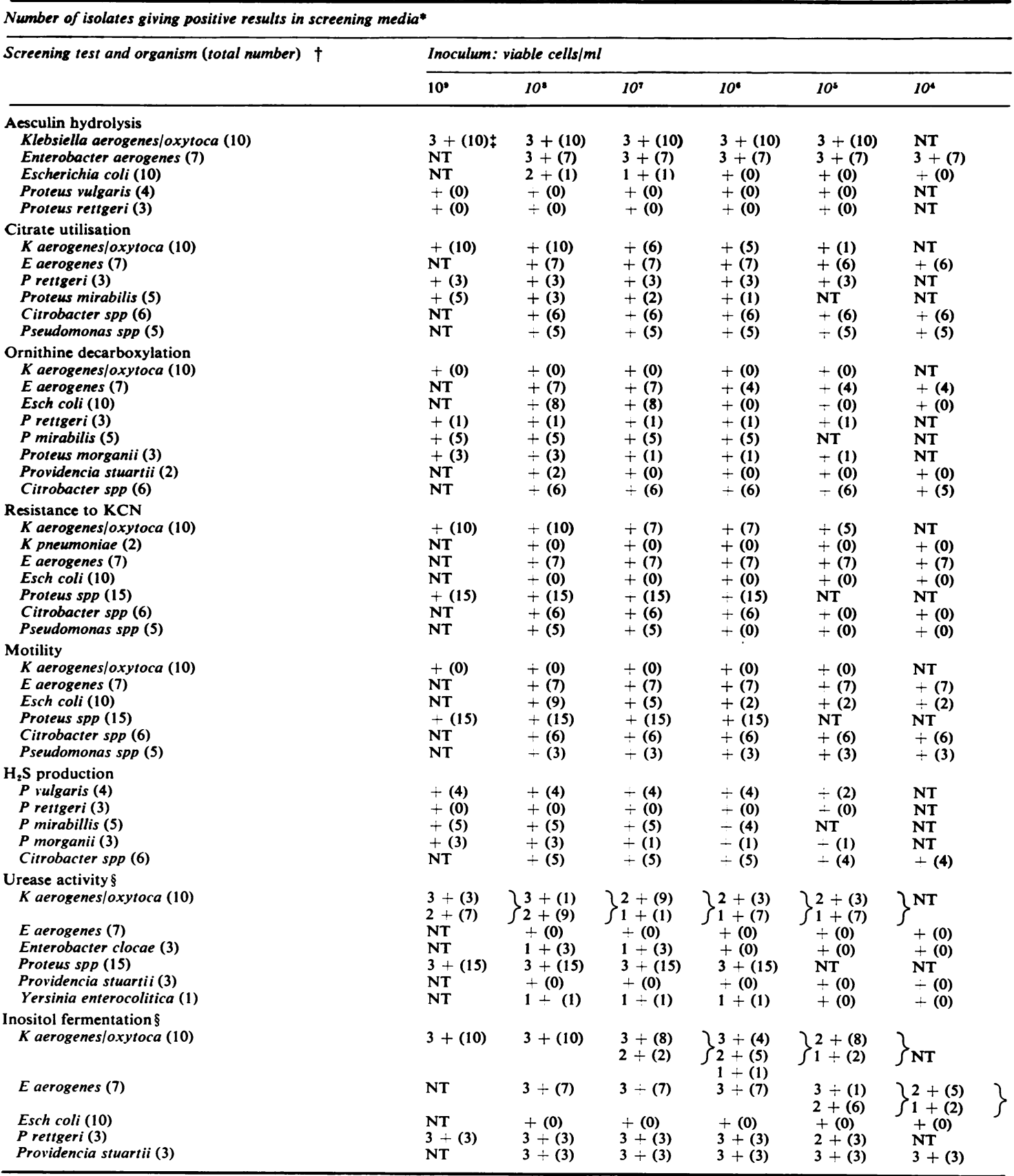

* Results recorded in semi-quantitative manner for aesculin hydrolysis, inositol fermentation, and urease activity, as described under Material and Methods.

†Total number of each species or genus evaluated for a specific test.

$¥$ Number of isolates (in parentheses) giving positive test reaction.

\$Different reactions given by isolates of Klebsiella aerogenes/oxytoca and Enterobacter aerogenes on urea and inositol media are recorded separately at each inoculum level.

NT $=$ not tested. 
Table 2 The forty major biotypic reaction patterns, ${ }^{*}$ on agar screening media, allowing immediate identification of 1529 clinical isolates of Enterobacteriaceae and Pseudomonas aeruginosa

\begin{tabular}{|c|c|c|c|c|c|c|c|c|c|c|c|c|c|c|c|}
\hline \multicolumn{16}{|c|}{ Biotype reaction patterns in screening media $\dagger$} \\
\hline \multirow[t]{2}{*}{ Organism } & \multicolumn{2}{|c|}{ No. of isolates } & \multirow[t]{2}{*}{$A$} & \multirow[t]{2}{*}{$C$} & \multirow[t]{2}{*}{$P P A$} & \multirow[t]{2}{*}{$O$} & \multirow[t]{2}{*}{$I$} & \multirow[t]{2}{*}{$X L D$} & \multirow[t]{2}{*}{$K C N$} & \multirow[t]{2}{*}{$\mathrm{H}_{2} \mathrm{~S}$} & \multirow[t]{2}{*}{ INOS } & \multirow[t]{2}{*}{$U$} & \multicolumn{2}{|l|}{$M$} & \multirow{2}{*}{$\begin{array}{l}\text { No. of } \\
\text { additional } \\
\text { biotype } \\
\text { reaction } \\
\text { patterns }\end{array}$} \\
\hline & Total & $\begin{array}{l}\text { No. with } \\
\text { specific } \\
\text { reaction } \\
\text { pattern }\end{array}$ & & & & & & & & & & & $\cdots$ & - & \\
\hline \multirow[t]{9}{*}{ Escherichia coli } & \multirow[t]{9}{*}{827} & $530 \ddagger$ & - & - & - & $\cdots$ & - & $\mathrm{Y}$ & - & - & - & - & 145 & 385 & \\
\hline & & $235 \ddagger$ & - & - & - & - & - & $\mathrm{Y}$ & - & - & - & - & 59 & 176 & \\
\hline & & $19 \ddagger$ & - & - & - & :- & - & $\mathbf{R}$ & - & - & - & - & 19 & 0 & \\
\hline & & $3 \ddagger$ & - & - & - & - & $\rightarrow$ & $\mathbf{R}$ & - & - & - & - & 3 & 0 & \\
\hline & & 7 & - & - & - & .. & $\ldots$ & NG & - & - & - & - & 0 & 7 & 0 \\
\hline & & 9 & - & - & - & - & - & NG & - & - & - & - & 0 & 9 & \\
\hline & & 10 & $2 \ldots$ & - & - & .. & - & $\mathrm{Y}$ & - & - & - & - & 9 & 1 & \\
\hline & & 5 & $2-$ & - & - & - & - & $\mathrm{Y}$ & - & - & - & - & 3 & 2 & \\
\hline & & 9 & - & - & - & .. & - & $\mathrm{Y}$ & - & - & 1. & - & 0 & 9 & \\
\hline \multirow{3}{*}{$\begin{array}{l}\text { Klebsiella aerogenes/ } \\
\text { edwardsii/atlantae }\end{array}$} & 191 & $141_{+}^{+}$ & 2 & .. & - & - & - & $\mathrm{Y}$ & - & - & 3 & $2-$ & 0 & 141 & \\
\hline & & 39 & $2-$ & - & - & - & - & $\mathrm{Y}$ & - & - & 3 & $1 \ldots$ & 0 & 39 & 3 \\
\hline & & $11 \S$ & - & $\ldots$ & - & - & - & $Y$ & - & - & 3 & $2-$ & 0 & 11 & \\
\hline Klebsiella oxytoca & 185 & $92 \ddagger$ & $2-$ & $\cdots$ & - & - & - & $\mathrm{Y}$ & . & - & 3 & 2 & 0 & 92 & \\
\hline & & 81 & 2 & - & - & - & - & $\mathrm{Y}$ & 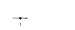 & - & 3 & $1-$ & 0 & 81 & 0 \\
\hline & & 12 & $2 \div$ & - & - & - & - & $\mathrm{Y}$ & - & - & 3. & - & 0 & 12 & \\
\hline Klebsiella pneumoniae & 3 & $2 \ddagger$ & $2-$ & - & - & - & - & $\mathrm{Y}$ & - & - & 1 . & $1-$ & 0 & 2 & \\
\hline & & 1 & $2-$ & - & - & - & - & $\mathrm{Y}$ & - & - & 2 . & $1-$ & 0 & 1 & 0 \\
\hline Klebsiella ozaenae & & & $2-$ & - & - & - & - & $\mathrm{Y}$ & - & - & 1 . & - & - & + & \\
\hline Enterobacter aerogenes & 81 & $77 \ddagger$ & $2-$ & . & - & - & - & $\mathbf{Y}$ & . & - & 3 - & - & 70 & 7 & \\
\hline & & 4 & $2-$ & - & - & .. & - & $\mathbf{R}$ & - & - & 3 . & - & 4 & 0 & 5 \\
\hline Serratia spp & 2 & $2^{\sigma}$ & $2-$ & - & - & - & . & $\ddot{Y}$ & - & - & 3 & $1-$ & 2 & 0 & 0 \\
\hline Citrobacter spp & 74 & $42 \ddagger$ & - & $\rightarrow$ & - & - & - & $\mathrm{Y}$ & . & -. & - & - & 7 & 35 & \\
\hline & & $25 \ddagger$ & - & . & - & - & - & $\dot{Y}$ & .. & - & - & - & 17 & 8 & \\
\hline & & $3+$ & - & - & - & .. & - & $\mathrm{Y}$ & .. & - & - & - & 0 & 3 & 5 \\
\hline & & 2 & - & .. & - & - & - & $\mathrm{Y}$ & . & - & - & - & 2 & 0 & \\
\hline & & 2 & - & + & - & - & - & B & . & - & - & - & 0 & 2 & \\
\hline Proteus mirabilis & 89 & $48 \ddagger$ & - & .. & $\ldots$ & & - & B & - & - & - & 3 . & 48 & 0 & \\
\hline & & 29 & - & - & - & . & ... & B & - & & - & $3-$ & 29 & 0 & 5 \\
\hline & & 12 & - & - & - & & $\cdots$ & $\mathrm{Y}$ & - & . & - & $3-$ & 12 & 0 & \\
\hline Proteus vulgaris & 12 & $6 \ddagger$ & - & ... & .. & - & - & $\mathrm{Y}$ & - & . & - & $3-$ & 6 & 0 & \\
\hline & & $4 \ddagger$ & - & - & - & - & - & $\mathrm{Y}$ & - & - & - & $3-$ & 4 & 0 & 3 \\
\hline & & 2 & $1-$ & - & - & - & - & $\mathrm{Y}$ & - & - & - & 3 & 2 & 0 & \\
\hline Proteus morganii & 10 & $10 \ddagger$ & - & - & - & & - & $\mathbf{R}$ & . & - & - & $3-$ & 9 & 1 & 1 \\
\hline Proteus rettgeri & 21 & $13 \ddagger$ & - & . & . & - & .. & $\mathrm{Y}$ & - & - & 3 . & 3 . & 9 & 4 & \\
\hline & & $6 \pm$ & $1 \ldots$ & - & - & - & - & $\mathrm{Y}$ & $\ldots$ & - & 3. & $3-$ & 2 & 4 & 4 \\
\hline & & $2^{+}$ & 2 & - & . & - & - & $\mathrm{Y}$ & - & - & 3 . & 3 - & 2 & 0 & \\
\hline Providencia stuartii & 5 & $5 \ddagger$ & - & - & - & - & -. & $\mathbf{R}$ & .. & - & 3. & - & 0 & 5 & 2 \\
\hline Providencia alcalifaciens & 4 & 4 & - & . & - & - & .. & $\mathbf{R}$ & - & - & - & - & 3 & 1 & 2 \\
\hline Yersinia enterocolitica & 3 & $3 \ddagger$ & - & - & - & - & -- & $\mathbf{R}$ & - & - & - & $1-$ & 3 & 0 & 0 \\
\hline Salmonella spp & & & - & $\because$ & - & . & - & $B / Y$ & - & $\therefore$ & - & - & $\cdots$ & - & \\
\hline Shigella spp & & & - & - & - & $=$ & \pm & $\mathrm{Y}$ & - & - & - & - & - & & \\
\hline Pseudonomas spp & 21 & $16 \ddagger$ & - & . & - & - & $=$ & $\mathbf{R}$ & & - & - & - & 9 & 7 & 3 \\
\hline & & 5 & $1 \div$ & - & - & - & - & $\mathbf{R}$ & - & - & - & - & 5 & 0 & \\
\hline
\end{tabular}

* Thirty-three other observed reaction patterns included 15 represented by a single isolate and 18 represented by more than one isolate but less than one per cent of the total isolates of species concerned. A further five reaction patterns could be resolved only after two further biochemical tests (Tables 3, 4).

+Biochemical patterns were established on the basis of aesculin hydrolysis (A); citrate utilisation (C); phenylalanine deaminase activity (PPA); $N$ ornithine decarboxylase activity $(\mathrm{O})$; indole production $(\mathrm{I})$; colony colour $(\mathbf{R}=$ red, $B=$ black, $\mathrm{Y}=$ yellow, NG $=$ no growth) on $\mathrm{xy}$ ylose $\mathrm{O}$ lysine deoxycholate agar (XLD); hydrogen sulphide production $\left(\mathrm{H}_{2} \mathrm{~S}\right)$ and inositol fermentation (INOS) in inositol-hydrogen sulphide-motility $\mathrm{N}$ medium; and urease activity (U). Motility of isolates of each reaction pattern were recorded as positive $(+)$ or negative $(-)$ on inositol-hydrogen $\bigcirc$ sulphide-motility medium at $37^{\circ} \mathrm{C}$. All other reactions were recorded as described in Materials and Methods.

tThe expected specific reaction patterns compiled from published reports on bacteria derived from culture collection and clinical sources. SOf a total of 11 isolates 8 were from patients in closely adjacent wards and represented repeated isolations from three different patients. Published results for Salmonella spp, Shigella spp, and Klebsiella ozaenae not included in this study of isolates from clinical material.

Three cultures include two atypical isolates of Serratia spp.

sielleae was little influenced by inoculum size and isolates of Enterobacter aerogenes showed rapid fermentation over a wide dilution range. The effect of inoculum size was most pronounced and of most importance among Klebsiella isolates. A fall in inositol fermentation paralleling a fall in inoculum size could be of importance in distinguishing Klebsiella aerogenes/oxytoca/edwardsii/atlantae, recorded elsewhere as consistently rapid fermenters, from slow fermenters such as $K$ ozaenae and $K$ pneumoniae (Barr and Mahood, 1976).

Our results showed the important effect of 
inoculum size on the results obtained in the biochemical tests described on screening media. So far as our experience goes, we do not know of any case in which the recording of a positive reaction at one inoculum level was accompanied by negative reactions at higher inoculum levels. The intrinsic error must be considered to be the recording, in the use of low inocula, of false negative results in 16-hour incubations. Similarly, even at the highest inoculum levels $\left(10^{9}\right.$ viable cells $\left./ \mathrm{ml}\right)$ insufficient turbidity is introduced to influence the recording of false positive resistance to potassium cyanide.

Indeed, within a wide range of inoculum size $\left(10^{9}-10^{7}\right.$ viable cells $/ \mathrm{ml}$, which is accompanied by a clearly visible reduction in turbidity and reduction in optimal density) the semi-quantitative recording of aesculin hydrolysis, inositol fermentation, and urease activity was influenced in few cases.

\section{EVALUATION OF SCREENING SCHEME IN}

ROUTINE USE

Normally the colony characteristics of the bacteria screened were observed on nutrient blood agar and MacConkey agar, the inoculum for screening media being derived from the MacConkey agar plate. This allowed purity of cultures to be established and lactose fermentation to be recorded.

A total of 1659 isolates were screened by the methods described. All isolates except 21 of Pseudomonas spp were oxidase-negative, Gram-negative bacilli. The reactions obtained gave a total of 78 distinguishable patterns, the major 45 patterns being shown in Tables 2,3 , and 4.

The reaction patterns expected for different species or genera on the basis of published reports are shown in Table 2 and confirmed with stock cultures in this study. The majority of isolates of each species, species group (for example, Klebsiella aerogenes/edwardsii/atlantae), or genus within the 1659 isolates investigated were identified by the characteristic patterns described. Thus, for example, 787 out of 827 isolates $(95.1 \%$ ) of $E$ coli, 180 out of 191 isolates $(94.2 \%)$ of $K$ aerogenes/edwardsii) atlantae, and 87 out of 132 isolates $(65.9 \%)$ of

Table 3 Resolution of biotype reaction patterns characteristic of species of Klebsielleae (Enterobacter aerogenes, E cloacae, Hafnia alvei, and Serratia marcescens) but not allowing immediate identification: tests to complete identification of some isolates

Biotype reaction patterns on screening media

\begin{tabular}{llllllllllllll}
\hline $\begin{array}{l}\text { Total } \\
\text { isolates }\end{array}$ & $A$ & $C$ & $P P A$ & $O$ & $I$ & $X L D$ & $K C N$ & $H_{2} S$ & INOS $U$ & $M$ & $\begin{array}{l}\text { Additional tests for } \\
\text { organism identification }\end{array}$ \\
\hline
\end{tabular}

$2++-\quad+\quad+\quad-\quad-\quad 204$

Arabinose fermenta-

* Numbers in parentheses = number of isolates ascribed to each species.

+Further tests not carried out.

Table 4 Resolution of biotype reaction patterns characteristic of Enterobacter cloacae, Hafnia alvei, or Citrobacter spp but not allowing immediate identification: tests to complete identification of some isolates

\begin{tabular}{|c|c|c|c|c|c|c|c|c|c|c|c|c|c|c|}
\hline \multicolumn{13}{|c|}{ Biotype reaction patterns on screening media } & \multirow{2}{*}{$\begin{array}{l}\text { Additional tests for } \\
\text {-organism identification }\end{array}$} & \multirow{2}{*}{ Identification } \\
\hline $\begin{array}{l}\text { Total } \\
\text { isolates }\end{array}$ & $A$ & $C$ & $P P A$ & $O$ & $I$ & $X L D$ & KCN & $\mathrm{H}_{2} \mathrm{~S}$ & INOS & $U$ & $\begin{array}{l}M \\
+\end{array}$ & - & & \\
\hline 7 & - & + & - & + & + & $\mathbf{R}$ & + & - & - & - & 5 & 2 & & $\begin{array}{l}\text { Citrobacter spp (5)* } \\
\text { Not identified } \dagger(2)\end{array}$ \\
\hline 20 & - & + & - & + & + & $\mathbf{Y}$ & + & - & - & - & 16 & 4 & $\begin{array}{l}\text { Methyl-red test, } \\
\text { lysine decarboxylase } \\
\text { test }\end{array}$ & $\begin{array}{l}\text { Citrobacter spp (12) } \\
\text { Not identified (8) }\end{array}$ \\
\hline 2 & - & + & - & + & - & $\mathbf{Y}$ & + & - & - & - & 2 & 0 & & $\begin{array}{l}\text { Enterobacter cloacae (1) } \\
\text { Citrobacterspp (1) }\end{array}$ \\
\hline
\end{tabular}

* Numbers in parentheses = number of isolates ascribed to each species.

†Further tests not carried out. 
Proteus spp gave patterns immediately recognisable from published reports of their biochemical activities.

The 45 major patterns described comprised 1584 isolates, $95.3 \%$ of the total number screened. Of these the 1529 isolates $(92.1 \%)$ listed in Table 2 were identified to genus or species level without further tests. The identification of a further 55 isolates to genus or species level was completed after further tests (Tables 3,4 ).

Three major biotypes typical of published reports of biochemical activity and 6 minor biotypic reaction patterns of $E$ coli were recorded. The minor biotypes included those showing aesculin hydrolysis and inositol fermentation already described (Barr and Mahood, 1977) and did not hinder identification. Within this scheme Yersinia enterocolitica is differentiated from $E$ coli on the basis of colour change on XLD agar and weak urease activity. Pasteurella pneumotropica is differentiated from $Y$ enterocolitica on the basis of oxidase activity.

Among the Klebsielleae the rapid inositol fermenters $K$ aerogenes, $K$ oxytoca, and $E$ aerogenes were clearly differentiated from one another and from other Klebsielleae-the incidence of $K$ oxytoca, $(49.2 \%)$ of Klebsiella isolates, being considerably higher than in previous reports (Davis and Matsen, 1974). Among other Klebsiella spp only K pneumoniae could otherwise be differentiated, although stock cultures of $K$ ozaenae could also be presumptively identified on the basis of slow inositol fermentation (Barr and Mahood, 1976). One novel biotype among the $K$ aerogenes/edwardsii/atlantae group was characterised by the absence of aesculin hydrolysis.

Differentiation of Enterobacter spp and Serratia spp was not achieved within the screening scheme. Some isolates of $E$ aerogenes lacked the ability to ferment inositol under the conditions described and could be differentiated from Enterobacter cloacae and Serratia marcescens only by further biochemical tests (Table 3) (Chadwick et al., 1974; Barr and Mahood, 1977).

Two novel isolates of Klebsielleae were also encountered in this investigation (Table 2). These comprised two rapid inositol-fermenting, lactose non-fermenting isolates of Serratia spp which were differentiated from other biotypes by the presence of tryptophanase activity.

Three biotype reaction patterns (Table 4) did not allow immediate differentiation between Enterobacter cloacae and Citrobacter spp. Two reaction patterns included indole positive reactions and were included here to confirm the absence of tryptophanase activity among isolates of $E$ cloacae. This was considered necessary in later stages of the screen when the presumptive presence of tryptophanase among other Klebsielleae had been established. As shown in Table 4, no indole-positive isolates of $\frac{\overline{0}}{-}$ Enterobacter cloacae were reported from this study. $\Rightarrow$

This scheme should, however, allow the differentiation of most Enterobacter spp and Serratia spp들 within 48 hours of isolation. The presence or absence $\frac{\overline{\bar{c}}}{\bar{s}}$ of lactose fermentation on primary isolation? MacConkey agar plates and the test results obtained $\propto$ on basic screening media within 16 hours should allow selection of further biochemical tests (arabin- $\vec{O}$ ose fermentation, lysine decarboxylation, and methylred test) to allow differentiation of genera and species $\vec{\omega}$ after a further 24 hours.

Reaction patterns given by eight major biotypes? of Citrobacter spp are shown in Tables 2 and 4, the biotypes described in Table 4 requiring to be furthero' tested by methyl-red and lysine decarboxylase tests $\overrightarrow{0}$ to ensure differentiation from $E$ cloacae. The major biotypes recorded (Table 2) had the properties? characteristic of the two species, Citrobacter $\vec{c}$ freundii and $C$. intermedius, recognised by Sedlák et al. (1971). The two major biotypes recognised in ${ }^{\Phi}$ Table 4 closely resembled $C$ intermedius, $C$ koseri, $\overrightarrow{0}$ and the genus Levinea described by Young et al.. (1971). However, other intergrading isolates are alsoo recorded, which could be expected from the number of species originally described by Werkman and Gillen (1932). An additional five minor biotypesฏ included other intergrading forms which produced $\mathbb{Q}$ indole and hydrogen sulphide either on XLD or $\Rightarrow$ inositol-hydrogen sulphide-motility medium. It has윽 been noted in Table 2 that Citrobacter spp and Salmonella spp are differentiated on the basis of sensitivity to potassium cyanide. Differentiation of Proteus spp and Providencia spp within the screeningo regimen was clearly defined. The inositol fermenting biotypes of Providencia stuartii (three in number) were clearly differentiated on this test alone fromo Providencia alcalifaciens.

Ten major and 13 minor biotype reaction patterns윽 were distinguished among isolates of Proteus spp.> All biotypes of Proteus rettgeri were readily differentiated from other Proteus spp on the basis of inositoln fermentation, and were distinguished from Providencia stuartii by virtue of colony colour on XLDO agar and strong urease activity.

Proteus morganii isolates gave the most consistent reaction pattern and were immediately recognisable Although no citrate positive isolates were reported here these would, should they arise, presumably.? readily be differentiated from indole-positive Proteus ${ }^{\top}$ mirabilis isolates on the basis of colony colour on XLD agar and the absence of $\mathrm{H}_{2} \mathrm{~S}$ production on? inositol- $\mathrm{H}_{2} \mathrm{~S}$-motility agar.

As would be expected from such a study, the largest number of Proteus isolates were ascribed ton the species $P$ mirabilis and to biotypes related to 
$\boldsymbol{P}$ vulgaris, species which have shown in numerical taxonomic studies to be closely related (McKell and Jones, 1976). Indeed, as shown in Tables 2, the presence of indole production among large numbers of $P$ mirabilis isolates $(46 \%)$, which has been previously noted by Matsen et al. (1972), variability in $\mathrm{H}_{2} \mathrm{~S}$ production by $\boldsymbol{P}$ mirabilis isolates on XLD agar, and variability in citrate utilisation by isolates of $\boldsymbol{P}$ vulgaris make it difficult on the basis of a narrow range of biochemical tests to ascribe some isolates in this group to particular species. However, although the presence of eight biotypes of $\boldsymbol{P}$ mirabilis and six biotypes of $\boldsymbol{P}$ vulgaris represent intergrading forms which in this screening scheme may defy definition, the recording of these biotypes in themselves is a valuable aid in epidemiological surveillance within this group.

Isolates of Pseudomonas spp were also included in the study because they constituted the most commonly isolated oxidase-positive Gram-negative bacillus, which Chadwick et al. (1974) indicated were clearly differentiated from other Gram-negative bacilli by a positive citrate reaction, a red colony colour on XLD agar, and negative results in other screening tests. We have confirmed these observations, included weak aesculin hydrolysis as a further criterion for identification in 16-hour incubations, and distinguished Pseudomonas aeruginosa by virtue of green pigmentation on phenylalanine deaminase agar.

\section{Discussion}

Forty-five major and 33 minor biotypic reaction patterns were recorded within this biochemical screening regimen for the identification of Enterobacteriaceae. The major patterns included those which, from published reports of the biochemical activities of different genera and species, would have been expected to predominate. The scheme allowed $92.1 \%$ of isolates to be identified to species level or, in the case of many Klebsiella spp and Citrobacter $s p p$, genus level on the basis of recognisable reaction patterns. A further $3.2 \%$ were identified as Citrobacter spp or as species of Klebsielleae after three further biochemical tests. The remaining $4.7 \%$ of isolates comprised those which have been ascribed to species or genera but whose reaction patterns were atypical or poorly represented as a percentage of isolates of the particular species or genera.

No attempt has been made to ascribe species names to isolates of Citrobacter or to introduce biochemical tests to further species definition among isolates of Klebsiella. Similarly, the limitations in differentiation between Proteus mirabilis and Proteus vulguris in the presence of so many isolates giving atypical reactions has been noted and the value of simple recording of biotypic reaction patterns, without attempting species definition, has been recognised.

The reproducibility of the screening tests was established by repeated testing with stock cultures and by the screening of multiple isolates of an organism from the same source on successive occasions. Indeed, by recognising biotypes it has often been possible to predict the source of a particular bacillus.

We have emphasised, however, that careful standardisation of techniques is required to obtain reproducible biotypic patterns. Of the possible variables we have considered inoculum size to be critical, the importance of standardisation being, as reported, more important in some tests than in others. But we do not know of any case in which a positive reaction at one inoculum level was accompanied by a negative reaction with the use of a higher inoculum. We therefore suggest that using a heavy inoculum (at least $10^{7}$ viable cells $/ \mathrm{ml}$ ), dispensed in the volume we used, should prevent the recording of false negative results in 16-hour incubations.

The system described is clearly adaptable to use with a multi-inoculator device, using replidishes as growth chambers, although it would then be necessary to ensure that a sufficiently high inoculum was delivered. By manual means, using Pasteur pipettes for inoculation, 50 cultures can be put through the system, with a total of two hours required for inoculum preparation, inoculation, and recording results.

We envisage that this system might provide a means for epidemiological surveillance of Enterobacteriaceae by biochemical typing. Result recording could easily be accommodated on a simple computer programme which could allow rapid retrieval of specific biotypic reaction patterns. The primary biotyping reaction scheme can easily be supplemented by biotyping schemes specific for particular genera or species.

We thank Mrs W. Booth for her energy and care in preparing the text.

\section{References}

Barr, J. G. and Mahood, R. J. (1976). An assessment of inositol fermentation by Klebsiellae and its implication in screening media. Journal of Clinical Pathology, 29, 827-832.

Barr, J. G. and Mahood, R. J. (1977). Complementary use of aesculin hydrolysis and inositol fermentation in the characterisation of Klebsielleae. Journal of Clinical Pathology, 30, 45-49.

Barritt, M. M. (1936). The intensification of the Voges- 
Proskauer reaction by the addition of $a$-naphthol. Journal of Pathology and Bacteriology, 42, 441-454.

Berger, U. and Einecke, H. (1973). Zur Unterscheidung der Gattungen Klebsiella-Enterobacter und ihrer Arten in der Routinediagnostik. Zentralblatt fur Bakteriologie, Parasitenkunde, Infektionskrankleiten und Hygiene Erst Abteilung: Originale, Reihe A: Medizinische Mikrobiologie und Parasitologie, 225, 471-486.

Carpenter, K. P., Lapage, S. P., and Steel, K. J. (1966). Biochemical identification of Enterobacteriaceae. In Identification Methods for Microbiologists, Part A, edited by B. M. Gibbs and F. A. Skinner, p. 21. Academic Press, London.

Chadwick, P., Delisle, G. J., and Byer, M. (1974). Biochemical identification of hospital enterobacteria by replica agar plating. Canadian Journal of Microbiology, 20, 1653-1664.

Christensen, W. B. (1946). Urea decomposition as a means of differentiating Proteus and paracolon cultures from each other and from Salmonella and Shigella types. Journal of Bacteriology, 52, 461-466.

Cowan, S. T. (1974). Cowan and Steel's Manual for the Identification of Medical Bacteria, 2nd edition. Cambridge University Press, London.

Davis, T. J. and Matsen, J. M. (1974). Prevalence and characteristics of Klebsiella species: relation to association with a hospital environment. Journal of Infectious Diseases, 130, 402-405.

Donovan, T. J. (1966). A Klebsiella screening medium. Journal of Medical and Laboratory Technology, 23, 194-197.

Edwards, P. R. and Ewing, W. H. (1962). Identification of Enterobacteriaceae, 2nd edition. Burgers Publishing Company, Minneapolis.

Fay, G. D. and Barry, A. L. (1972). Rapid ornithine decarboxylase test for the identification of Enterobacteriaceae. Applied Microbiology, 23, 710-713.

Lassen, J. (1975). Rapid identification of Gram-negative rods using a three-tube method combined with a dichotomic key. Acta Pathologica et Microbiologica Scandinavica, 83, (6) Supplement, 525-533.

McKell, J. and Jones, D. (1976). A numerical taxonomic study of Proteus-Providence bacteria. Journal of Applied Bacteriology, 41, 143-161.

Matsen, J. M., Blazevic, D. J., Ryan, J. A., and Ewing,
W. H. (1972). Characterization of indole-positive 음 Proteus mirabilis. Applied Microbiology, 23, 592-594.

Miles, A. A. and Misra, S. S. (1938). The estimation of the bactericidal power of the blood. Journal of Hygiene, 38, 732-748.

Møller, V. (1955). Simplified tests for some amino-acid decarboxylases and for the arginine dihydrolase system. Acta Pathologica et Microbiologica Scandinavica, 36, 158-172.

Nord, C. E., Wadström, T., and Dahlbäck, A. (1975). Evaluation of different diagnostic kits for Enterobacteriaceae. In New Approaches to the Identification of Micro-organisms, edited by C. G. Héden and T. Illeni, pp. 393-406. John Wiley.

Rogers, K. B. and Taylor, J. (1961). Laboratory diagnosis of gastroenteritis due to Escherichia coli. Bulletin of the World Health Organisation, 24, 59-71.

Rosner, R. F. (1970). Identification of enteric bacilli directly from primary isolation media using reagentimpregnated paper strips. American Journal of Clinical Pathology, 54, 587-595.

Sedlák, J., Puchmayerová-Slajsová, M., Keleti, J., and Lüderitz, O. (1971). On the taxonomy, ecology and immunochemistry of genus, Citrobacter. Journal of Hygiene, Epidemiology, Microbiology and Immunology, 15, 366-374.

Taylor, W. I. (1969). Isolation of Shigellae. I. Xyloselysine agars; new media for isolation of enteric pathogens. American Journal of Clinical Pathology, 44, 471-475.

Tomfohrde, K. M., Rhoden, D. L., Smith, P. B., and Balows, A. (1973). Evaluation of the Redesigned Enterotube - a system for the identification of Enterobacteriaceae. Applied Microbiology, 25, 301-304.

Wasilauskas, B. L. (1971). Preliminary observations on the rapid differentiation of the Klebsiella-EnterobacterSerratia group on bile-esculin-agar. Applied Microbiology, 21, 162-163.

Werkman, C. H. and Gillen, G. F. (1932). Bacteria producing trimethylene glycol. Journal of Bacteriology, 23, 167-182.

Young, V. M., Kenton, D. M., Hobbs, B. J., and Moody, M. R. (1971). Levinea, a new genus of the family Enterobacteriaceae. International Jounnal of Systematic Bacteriology, 21, 58-63. 